\title{
Growth of regulators in finite abelian coverings
}

THANG T Q LÊ

\begin{abstract}
We show that the regulator, which is the difference between the homology torsion and the combinatorial Ray-Singer torsion, of finite abelian coverings of a fixed complex has sub-exponential growth rate.
\end{abstract}

54H20; 57Q10, 37B50, 37B10

\section{Introduction}

\subsection{Based free complex over group ring and its quotients}

Suppose $\pi$ is a finitely presented group and $\mathbb{Z}[\pi]$ is the group ring of $\pi$ over the ring $\mathbb{Z}$ of integers.

Let $\mathcal{C}$ be a finitely generated based free $\mathbb{Z}[\pi]$-complex

$$
0 \rightarrow C_{m} \stackrel{\partial_{m}}{\longrightarrow} C_{m-1} \stackrel{\partial_{m-1}}{\longrightarrow} C_{m-2} \longrightarrow \cdots \stackrel{\partial_{2}}{\longrightarrow} C_{1} \stackrel{\partial_{1}}{\longrightarrow} C_{0} \rightarrow 0 .
$$

Here "based free" means each $C_{k}$ is a free $\mathbb{Z}[\pi]$-module equipped with a preferred base.

For a normal subgroup $\Gamma \triangleleft \pi$ let $\mathcal{C}_{\Gamma}:=\mathbb{Z}[\pi / \Gamma] \otimes_{\mathbb{Z}[\pi]} \mathcal{C}$. Assume that the index $[\pi: \Gamma]$ is finite. Then $\mathcal{C}_{\Gamma}$ is a finitely generated based free $\mathbb{Z}$-complex, where the preferred base of $\mathbb{Z}[\pi / \Gamma] \otimes_{\mathbb{Z}[\pi]} C_{k}$ is defined using the one of $C_{k}$ in a natural way.

A prototypical case is the following. Suppose $\tilde{X} \rightarrow X$ is a regular covering with $\pi$ the group of deck transformations and $X$ a finite CW-complex. Choose a lift in $\tilde{X}$ of each cell of $X$. Then the $\mathrm{CW}$-complex $\mathcal{C}$ of $\tilde{X}$ induced from that of $X$ is a finitely generated based free $\mathbb{Z}[\pi]$-complex. For a normal subgroup $\Gamma \triangleleft \pi, \mathcal{C}_{\Gamma}$ is the $\mathrm{CW}$-complex of the covering $X_{\Gamma}$, corresponding to the group $\Gamma$, and $H_{k}\left(\mathcal{C}_{\Gamma}\right)$ is the $k^{\text {th }}$ homology of the covering $X_{\Gamma}$. Usually, interesting invariants do not depend on the choice of the lifts of cells of $X$. 


\subsection{Two torsions}

We can define two torsions of the quotient complex $\mathcal{C}_{\Gamma}$, the homology torsion $\tau^{H}\left(\mathcal{C}_{\Gamma}\right)$ and the combinatorial Ray-Singer torsion $\tau^{R S}\left(\mathcal{C}_{\Gamma}\right)$, as follows. The homology torsion is

$$
\tau^{H}\left(\mathcal{C}_{\Gamma}\right):=\left(\prod_{k}^{*}\left|\mathfrak{t o r}_{\mathbb{Z}}\left(H_{k}\left(\mathcal{C}_{\Gamma}\right)\right)\right|\right)^{-1} \in \mathbb{R}_{+},
$$

where $\operatorname{tor}_{\mathbb{Z}}(M)$ is the $\mathbb{Z}$-torsion part of the finitely generated abelian group $M$, and $\prod_{k}^{*} a_{k}$ is the alternating product

$$
\prod_{k}^{*} a_{k}=\prod_{k} a_{k}^{(-1)^{k}}
$$

The Ray-Singer torsion of $\mathcal{C}_{\Gamma}$ is

$$
\tau^{R S}\left(\mathcal{C}_{\Gamma}\right)=\prod_{k}^{*} \operatorname{det}^{\prime}\left(\partial_{k}\right) \in \mathbb{R}_{+}
$$

where $\operatorname{det}^{\prime}$ is the geometric determinant of linear maps between based Hermitian spaces. We recall the definition of $\operatorname{det}^{\prime}$ in Section 2.

\subsection{Comparison: general question}

We want to compare the asymptotics of the two torsions as $\Gamma$ becomes "thinner and thinner in $\pi$ ", so that $\pi / \Gamma$ approximates $\pi$ in the following sense. A finite set $S$ of generators of $\pi$ defines a word length function $l_{S}$ (and hence a metric) on $\pi$. Define

$$
\langle\Gamma\rangle:=\min \left\{l_{S}(x) \mid x \in \Gamma \backslash\{e\}\right\} .
$$

Here $e$ is the unit of $\pi$. In all that follows, statements do not depend on the choice of the generator set $S$, since the metrics of two different generator sets are quasi-isometric.

We are interested in the following question: Suppose $\mathcal{C}$ is $L^{2}$-acyclic (see eg Lück [12]). Under what conditions does it hold that

$$
\lim _{\substack{\langle\Gamma\rangle \rightarrow \infty,|\pi: \Gamma|<\infty}} \frac{\ln \left(\tau^{H}\left(\mathcal{C}_{\Gamma}\right)\right)-\ln \left(\tau^{R S}\left(\mathcal{C}_{\Gamma}\right)\right)}{|\pi: \Gamma|}=0 ?
$$

The motivation of this question comes from the question [12]: can one approximate $L^{2}$-torsions by finite-dimensional analogs? In some favorable conditions, one expects that the growth rate of each of $\tau^{H}$ and $\tau^{R S}$ is the $L^{2}$-torsion, hence they must be the same. 
Remark 1.1 (a) If $\left\{\Gamma_{n}, n=1,2, \ldots\right\}$ is a sequence of exhausting nested normal subgroups of $\pi$, ie, $\Gamma_{n+1} \subset \Gamma_{n}$ and $\bigcap_{n} \Gamma_{n}=\{e\}$, then $\lim _{n \rightarrow \infty}\left\langle\Gamma_{n}\right\rangle=\infty$. The limit in (1) is more general (stronger) than the limit of an exhausting nested sequence, as we do not have the "nested" property.

(b) There exists a sequence $\Gamma_{n} \triangleleft \pi$ such that $\lim _{n \rightarrow \infty}\left\langle\Gamma_{n}\right\rangle=\infty$ if and only if $\pi$ is residually finite. Hence, the left hand side of (1) makes sense only when $\pi$ is residually finite.

(c) Define $\operatorname{tr}_{\pi}(x)=\delta_{x, e}$ for $x \in \pi$. This functional trace is the base for the definition of many combinatorial $L^{2}$-invariants. For a fixed $x \in \pi$, we have

$$
\lim _{\langle\Gamma\rangle \rightarrow \infty} \operatorname{tr}_{\pi / \Gamma}(x)=\operatorname{tr}_{\pi}(x) .
$$

This is the reason why one expects that as $\langle\Gamma\rangle \rightarrow \infty$, many $L^{2}$-invariants (under some technical conditions) can be approximated by the corresponding invariants of $\pi / \Gamma$.

\subsection{Main results}

The main result of the paper treats the case $\pi=\mathbb{Z}^{n}$.

Theorem 1 Suppose $\mathcal{C}$ is an $L^{2}$-acyclic finitely generated based free $\mathbb{C}\left[\mathbb{Z}^{n}\right]$-complex. Then (1), with $\pi=\mathbb{Z}^{n}$, holds true.

We will not give the definition of $L^{2}$-acyclicity. Instead, for $\pi=\mathbb{Z}^{n}$, we will use an equivalent definition (Elek [4], Lück [12]): the $L^{(2)}$ homology $H_{k}^{(2)}(\mathcal{C})$ vanishes if and only if $H_{k}\left(\mathcal{C} \otimes_{\mathbb{Z}\left[\mathbb{Z}^{n}\right]} F\right)=0$. Here $F$ is the fractional field of the commutative domain $\mathbb{Z}\left[\mathbb{Z}^{n}\right]$.

Remark 1.2 (a) Our result does not imply that

$$
\lim _{\substack{\langle\Gamma\rangle \rightarrow \infty \\|\pi: \Gamma|<\infty}} \frac{\ln \left(\tau^{H}\left(\mathcal{C}_{\Gamma}\right)\right)}{|\pi: \Gamma|}=\lim _{\substack{\langle\Gamma\rangle \rightarrow \infty,|\pi: \Gamma|<\infty}} \frac{\ln \left(\tau^{R S}\left(\mathcal{C}_{\Gamma}\right)\right)}{|\pi: \Gamma|},
$$

as we cannot prove the existence of each of the limits. For $\pi=\mathbb{Z}$, it was known that both limits exist and are equal to the $L^{2}$-torsion of $\mathcal{C}$; see González-Acuña and Short [6], Riley [17], and Lück [12]. Even for the case where $\pi=\mathbb{Z}^{2}$ and $\mathcal{C}$ is a 2-term complex $0 \rightarrow C_{1} \rightarrow C_{0} \rightarrow 0$ (so that only $H_{0}(\mathcal{C})$ is non-trivial), there is still no proof of the conjecture that the $L^{2}$-torsion is equal to either of the above limits. For results and discussions of this and related conjectures, see Lück [12; 11], Bergeron and Venkatesh [1], Lê [10; 9], Friedl and Jackson [5], and Silver and Williams [20]. 
(b) It should be noted that the exact calculation of the torsion part of the homology of finite coverings, even in the abelian case, is very difficult; see Hillman and Sakuma [7], Mayberry and Murasugi [13], and Porti [15] for some partial results.

\subsection{Refinement}

Suppose $\pi$ is residually finite and the $L^{2}$-homology $H_{k}^{(2)}(\mathcal{C})=0$ for some $k$. For any normal subgroup $\Gamma \triangleleft \pi$ of finite index, the homology group $H_{k}\left(\mathcal{C}_{\Gamma}\right)$ is a finitely generated abelian group. Because $H_{k}^{(2)}(\mathcal{C})=0$ one should expect that $H_{k}\left(\mathcal{C}_{\Gamma}\right)$ is negligible. In fact, a theorem of Lück [11] (and Kazhdan for this case) says that

$$
\lim _{\substack{\langle\Gamma\rangle \rightarrow \infty \\|\pi: \Gamma|<\infty}} \frac{\mathrm{rk}_{\mathbb{Z}} H_{k}\left(\mathcal{C}_{\Gamma}\right)}{|\pi: \Gamma|}=0 .
$$

This means the free part $H_{k}\left(\mathcal{C}_{\Gamma}\right)_{\text {free }}$ of $H_{k}\left(\mathcal{C}_{\Gamma}\right)$ is small compared to the index. There is another measure of the free part $H_{k}\left(\mathcal{C}_{\Gamma}\right)_{\text {free }}$, denoted by $R_{k}\left(\mathcal{C}_{\Gamma}\right)$ and called the regulator, or volume; see [1] and Section 3. Another expression of the fact that $H_{k}\left(\mathcal{C}_{\Gamma}\right)_{\text {free }}$ is small compared to the index is expressed in the following statement, which complements the result of Kazhdan and Lück.

Theorem 2 Suppose $\mathcal{C}$ is a finitely generated based free $\mathbb{Z}[\pi]$-complex with $\pi=\mathbb{Z}^{n}$ and $H_{k}^{(2)}(\mathcal{C})=0$ for some index $k$. Then

$$
\lim _{\substack{\langle\Gamma\rangle \rightarrow \infty,|\pi: \Gamma|<\infty}} \frac{\ln \operatorname{vol}\left(H_{k}\left(\mathcal{C}_{\Gamma}\right)_{\text {free }}\right)}{|\pi: \Gamma|}=0 .
$$

Remark 1.3 The question (and some form of the conjecture) about the growth rate of regulators was first raised in [1], where, among other things, a special case of Theorem 2 was established: It was proved that if $\pi=\mathbb{Z}^{n}$ and $\Gamma$ runs the set of sublattices of the form $k \mathbb{Z}^{n}$, then (3) holds. The proof there can be modified to include the case when $\Gamma$ runs the set of uniform sublattices, as defined in [16]. Our result removes any restriction on $\Gamma$.

\subsection{On the proofs}

For the proofs we use tools in commutative algebra and algebraic geometry. In particular, we make essential use of the theory of torsion points in $\mathbb{Q}$-algebraic set (a simple version of the Manin-Mumford principle). We hope that the methods and results can be adapted to the case of elementary amenable groups. 


\section{Acknowledgements}

I would like to thank M Baker, N Bergeron, H Dao, W Lück, A Thom, U Zannier and the anonymous referee for helpful discussions/comments. The author is supported in part by an NSF grant.

\subsection{Organization of the paper}

In Section 2 we recall the notions of geometric determinant and volume. We discuss the relation between homology and Ray-Singer torsions in Section 3. An overview of the theory of torsion points in algebraic set is given in Section 4. Section 5 contains a crucial growth estimate which is needed in the proofs of the main theorems, given in Section 6.

\section{Geometric determinant, lattices and volume in based Her- mitian spaces}

In this section we recall the definition of geometric determinant and basic facts about volumes of lattices in based Hermitian spaces.

\subsection{Geometric determinant}

For a linear map $f: V_{1} \rightarrow V_{2}$, where each $V_{i}$ is a finite-dimensional Hermitian space, the geometric determinant $\operatorname{det}^{\prime}(f)$ is the product of all non-zero singular values of $f$. Recall that $x \in \mathbb{R}$ is singular value of $f$ if $x \geq 0$ and $x^{2}$ is an eigenvalue of $f^{*} f$. By convention, $\operatorname{det}^{\prime}(f)=1$ if $f$ is the 0 map. Thus we always have $\operatorname{det}^{\prime}(f)>0$.

Since the maximal singular value of $f$ is the norm $\|f\|$, we have

$$
\operatorname{det}^{\prime}(f) \leq\|f\|^{\operatorname{dim} V_{2}} \quad \text { if } f \text { is non-zero. }
$$

Remark 2.1 The geometric meaning of $\operatorname{det}^{\prime} f$ is the following. The map $f$ restricts to a linear isomorphism $f^{\prime}$ from $\operatorname{Im}\left(f^{*}\right)$ to $\operatorname{Im}(f)$; each is a Hermitian space. Then $\operatorname{det}^{\prime} f=\left|\operatorname{det}\left(f^{\prime}\right)\right|$, where the ordinary determinant $\operatorname{det}\left(f^{\prime}\right)$ is calculated using orthonormal bases of the Hermitian spaces.

\subsection{Based Hermitian space and volume}

Suppose $W$ is a finite-dimensional based Hermitian space, ie, a $\mathbb{C}$-vector space equipped with an Hermitian product $(\cdot, \cdot)$ and a preferred orthonormal basis. The $\mathbb{Z}$-submodule $\Omega \subset W$ spanned by the basis is called the fundamental lattice. 
For a $\mathbb{Z}$-submodule (also called a lattice) $\Lambda \subset W$ with $\mathbb{Z}$-basis $v_{1}, \ldots, v_{l}$, define

$$
\operatorname{vol}(\Lambda)=\left|\operatorname{det}\left(\left(v_{i}, v_{j}\right)_{i, j=1}^{l}\right)\right|^{1 / 2} \text {. }
$$

By convention, the volume of the 0 space is 1 . If $\Lambda \subset \Omega$, we say that $\Lambda$ is an integral lattice. It is clear that $\operatorname{vol}(\Lambda) \geq 1$ if $\Lambda$ is an integral lattice.

For a $\mathbb{C}$-subspace $V \subset W$, the lattice $V^{(\mathbb{Z})}:=V \cap \Omega$ is called the $\mathbb{Z}$-support of $V$. We define

$$
\operatorname{vol}(V):=\operatorname{vol}\left(V^{(\mathbb{Z})}\right)
$$

A lattice $\Lambda \subset \Omega$ is primitive if is cut out from $\Omega$ by some subspace, ie, $\Lambda=V^{(\mathbb{Z})}$ for some subspace $V \subset W$. By definition, any primitive lattice is integral.

As usual, we say that a subspace $V \subset W$ is defined over $\mathbb{Q}$ if it is defined by some linear equations with rational coefficients (using the coordinates in the preferred base). It is easy to see that $V$ is defined over $\mathbb{Q}$ if and only if it is spanned by its $\mathbb{Z}$-support. Suppose $V_{1}, V_{2}$ are subspaces of $W$ defined over $\mathbb{Q}$, and $f: V_{1} \rightarrow V_{2}$ is a $\mathbb{C}$-linear map. We say that $f$ is integral if $f\left(V_{1}^{(\mathbb{Z})}\right) \subset V_{2}^{(\mathbb{Z})}$.

We summarize some well-known properties of volumes of lattices (see eg Bertrand [2]).

Proposition 2.1 Suppose $V_{1}, V_{2}$ are subspaces of $W$ defined over $\mathbb{Q}$ of a based Hermitian space $W$ and $f: V_{1} \rightarrow V_{2}$ is an integral, non-zero $\mathbb{C}$-linear map. Then

$$
\begin{aligned}
\operatorname{vol}\left(V_{1}+V_{2}\right) & \leq \operatorname{vol}\left(V_{1}\right) \operatorname{vol}\left(V_{2}\right), \\
\operatorname{vol}(\operatorname{ker} f) \operatorname{vol}\left[f\left(V_{1}^{(\mathbb{Z})}\right)\right] & =\operatorname{det}^{\prime}(f) \operatorname{vol}\left(V_{1}\right) .
\end{aligned}
$$

For a detailed discussion of (6) and its generalizations to lattices in $\mathbb{Z}\left[\mathbb{Z}^{n}\right]$, see Raimbault [16].

\section{Regulator, homology torsion and Ray-Singer torsion}

In this section we explain the relation between the homology torsion and the combinatorial Ray-Singer torsion. Proposition 3.1 of this section will be used in the proof of main theorems.

Throughout this section we fix a finitely generated based free $\mathbb{Z}$-complex $\mathcal{E}$

$$
0 \rightarrow E_{m} \stackrel{d_{m-1}}{\longrightarrow} E_{m-1} \stackrel{d_{m-1}}{\longrightarrow} E_{m-2} \longrightarrow \cdots \stackrel{d_{2}}{\longrightarrow} E_{1} \stackrel{d_{1}}{\longrightarrow} E_{0} \rightarrow 0 .
$$

Define a Hermitian product on $E_{k} \otimes_{\mathbb{Z}} \mathbb{C}$ such that the preferred base is an orthonormal base. Now $E_{k} \otimes_{\mathbb{Z}} \mathbb{C}$ becomes a based Hermitian space. 
We use the notation

$$
Z_{k}=\operatorname{ker} d_{k}, \quad B_{k}=\operatorname{Im} d_{k+1}, \quad \bar{B}_{k}=\left(B_{k} \otimes_{\mathbb{Z}} \mathbb{C}\right) \cap E_{k} .
$$

Let $d_{k}^{*}: E_{k-1} \rightarrow E_{k}$ be adjoint of $d_{k}$ and $D_{k}: E_{k} \rightarrow E_{k}$ be defined by

$$
D_{k}=d_{k}^{*} d_{k}+d_{k+1} d_{k+1}^{*} .
$$

\subsection{Ray-Singer torsion and homology torsion}

Define the Ray-Singer torsion and the homology torsion of $\mathcal{E}$ by

$$
\begin{aligned}
\tau^{R S}(\mathcal{E}) & =\prod_{k}^{*} \operatorname{det}^{\prime}\left(d_{k}\right) \in \mathbb{R}_{+}, \\
\tau^{H}(\mathcal{E}) & =\left(\prod_{k}^{*}\left|\mathfrak{t o r}_{\mathbb{Z}}\left(H_{k}(\mathcal{E})\right)\right|\right)^{-1} .
\end{aligned}
$$

Remark 3.1 The Ray-Singer torsion and the homology torsion can be defined through the classical Reidemeister torsion as follows.

Let $\tilde{\mathfrak{h}}_{k}$ be an orthonormal basis of $\operatorname{ker}\left(D_{k}\right) \otimes_{\mathbb{Z}} \mathbb{C}=H_{k}\left(\mathcal{E} \otimes_{\mathbb{Z}} \mathbb{C}\right)$. With the bases $\left\{\tilde{\mathfrak{h}}_{k}\right\}$ of the homology of $\mathcal{E} \otimes_{\mathbb{Z}} \mathbb{C}$, one can define the Reidemeister torsion $\tau^{R}\left(\mathcal{E} \otimes_{\mathbb{Z}} \mathbb{C},\left\{\tilde{\mathfrak{h}}_{k}\right\}\right)$, defined up to signs (see eg Turaev [21]). It is not difficult to show that

$$
\tau^{R S}(\mathcal{E})=\left|\tau^{R}\left(\mathcal{E} \otimes_{\mathbb{Z}} \mathbb{C},\left\{\tilde{\mathfrak{h}}_{k}\right\}\right)\right| .
$$

Both $\bar{B}_{k}$ and $Z_{k}$ are primitive lattices in $E_{k}$, and $\bar{B}_{k} \subset Z_{k}$. There is a collection $\mathfrak{h}_{k}$ of elements of $Z_{k} \subset E_{k}$ that descend to a basis of the group $Z_{k} / \bar{B}_{k}$, the free part of $H_{k}(\mathcal{E})$. Since $\mathfrak{h}_{k}$ is a basis of $H_{k}\left(\mathcal{E} \otimes_{\mathbb{Z}} \mathbb{C}\right)$, the Reidemeister torsion $\tau^{R}\left(\mathcal{E} \otimes_{\mathbb{Z}} \mathbb{C},\left\{\mathfrak{h}_{k}\right\}\right)$ is defined. It is not difficult to prove the following generalization of the Milnor-Turaev formula $[14 ; 21]$ :

$$
\tau^{H}(\mathcal{E})=\left|\tau^{R}\left(\mathcal{E} \otimes_{\mathbb{Z}} \mathbb{C},\left\{\mathfrak{h}_{k}\right\}\right)\right|
$$

\subsection{Regulators}

By definition, $H_{k}(\mathcal{E})=Z_{k} / B_{k}$. The $\mathbb{Z}$-torsion of $H_{k}(\mathcal{E})$ is $\bar{B}_{k} / B_{k}$, and the free part $H_{k}(\mathcal{E})_{\text {free }}$ is isomorphic to $Z_{k} / \bar{B}_{k}$. For this reason, we define the volume $\operatorname{vol}\left(H_{k}(\mathcal{E})_{\text {free }}\right)$ to be

$$
R_{k}(\mathcal{E}):=\frac{\operatorname{vol}\left(Z_{k}\right)}{\operatorname{vol}\left(\bar{B}_{k}\right)}
$$


Here we follow the notation of [1], where $R_{k}$ is called the regulator. Using Identity (6), one can prove (see [1, Formula 2.2.4])

$$
\tau^{R S}(\mathcal{E})=\tau^{H}(\mathcal{E})\left(\prod_{k}^{*} R_{k}(\mathcal{E})\right) .
$$

We will use the following estimate of the regulator.

Proposition 3.1 Let $\widetilde{R}_{k}:=\operatorname{vol}\left(\operatorname{ker} D_{k}\right)$. For every $k$, one has

$$
\widetilde{R}_{k} \geq R_{k} \geq \frac{1}{\widetilde{R}_{k}}
$$

Proof Let $W$ be the orthogonal complement of $B_{k} \otimes_{\mathbb{Z}} \mathbb{C}$ in $Z_{k} \otimes_{\mathbb{Z}} \mathbb{C}$ and $p: Z_{k} \otimes_{\mathbb{Z}}$ $\mathbb{C} \rightarrow W$ be the orthogonal projection. Then

$$
R_{k}=\operatorname{vol}\left(p\left(Z_{k}\right)\right) \text {. }
$$

By Hodge theory (for finitely generated $\mathbb{Z}$-complexes),

$$
\operatorname{ker}\left(D_{k}\right)=E_{k} \cap W=W^{(\mathbb{Z})} .
$$

It follows that $\operatorname{ker}\left(D_{k}\right) \subset p\left(Z_{k}\right)$, and hence $\operatorname{vol}\left(p\left(Z_{k}\right)\right) \leq \operatorname{vol}\left(\operatorname{ker}\left(D_{k}\right)\right)$, or

$$
R_{k} \leq \widetilde{R}_{k}
$$

By [2, Proposition 1(ii)],

$$
R_{k}=\frac{\operatorname{vol}\left(Z_{k}\right)}{\operatorname{vol}\left(\bar{B}_{k}\right)}=\frac{\left[\left(W \cap Z_{k}^{*}\right): Z_{k}\right]}{\widetilde{R}_{k}},
$$

where $Z_{k}^{*}$ is the $\mathbb{Z}$-dual of $Z_{k}$ in $Z_{k} \otimes_{\mathbb{Z}} \mathbb{C}$ under the inner product. Note that $Z_{k}^{*}$ is also the orthogonal projection of $E_{k}$ onto $Z_{k} \otimes_{\mathbb{Z}} \mathbb{C}$.

Since the numerator of (9) is $\geq 1$, we have $R_{k} \geq 1 / \widetilde{R}_{k}$, which, together with (8), proves the proposition.

\section{Abelian groups, algebraic subgroups of $\left(\mathbb{C}^{*}\right)^{n}$ and torsion points}

We review some facts about representation theory of finite abelian groups in Section 4.1 and the theory of torsion points on rational algebraic sets (a simple version of ManinMumford principle) in Sections 4.2 and 4.3. 


\subsection{Decomposition of the group ring of a finite abelian group}

Suppose $A$ is a finite abelian group. The group ring $\mathbb{C}[A]$ is an $A$-module (the regular representation) and is a $\mathbb{C}$-vector space of dimension $|A|$. Equip $\mathbb{C}[A]$ with a Hermitian product so that $A$ is an orthonormal basis. This makes $\mathbb{C}[A]$ a based Hermitian space, with $\mathbb{Z}[A]$ the fundamental lattice.

Let $\hat{A}=\operatorname{Hom}\left(A, \mathbb{C}^{*}\right)$, known as the Pontryagin dual of $A$, be the group of all characters of $A$. Here $\mathbb{C}^{*}$ is the multiplicative group of non-zero complex numbers. We have $|\widehat{A}|=|A|$.

The theory of representations of $A$ over $\mathbb{C}$ is easy: $\mathbb{C}[A]$ decomposes as a direct sum of mutually orthogonal one-dimensional $A$-modules:

$$
\mathbb{C}[A]=\bigoplus_{\chi \in \widehat{A}} \mathbb{C} e_{\chi},
$$

where $e_{\chi}$ is the idempotent

$$
e_{\chi}=\frac{1}{|A|} \sum_{a \in A} \chi\left(a^{-1}\right) a .
$$

The vector subspaces $\mathbb{C} e_{\chi}$ are not only orthogonal with respect to the Hermitian structure, but also orthogonal with respect to the ring structure in the sense that $e_{\chi} e_{\chi^{\prime}}=$ 0 if $\chi \neq \chi^{\prime}$. Each $\mathbb{C} e_{\chi}$ is an ideal of the ring $\mathbb{C}[A]$.

From the trace identity (see eg Serre [19, Section 2.4]) we have, for every $a \in A$,

$$
\sum_{\chi \in \hat{A}} \chi(a)= \begin{cases}0 & \text { if } a \neq e \\ |A| & \text { if } a \neq e .\end{cases}
$$

Here $e \in A$ is the trivial element.

\subsection{Algebraic subgroups of $\left(\mathbb{C}^{*}\right)^{n}$ and lattices in $\mathbb{Z}^{n}$}

4.2.1 Algebraic subgroups of $\left(\mathbb{C}^{*}\right)^{n} \quad$ An algebraic subgroup of $\left(\mathbb{C}^{*}\right)^{n}$ is a subgroup that is closed in the Zariski topology.

For a lattice $\Lambda$, ie, a subgroup $\Lambda$ of $\mathbb{Z}^{n}$, not necessarily of maximal rank, let $G(\Lambda)$ be the set of all $\mathbf{z} \in \mathbb{C}^{n}$ such that $\mathbf{z}^{\mathbf{k}}=1$ for every $\mathbf{k} \in \Lambda$. Here for $\mathbf{k}=\left(k_{1}, \ldots, k_{n}\right) \in \mathbb{Z}^{n}$ and $\mathbf{z}=\left(z_{1}, \ldots, z_{n}\right) \in\left(\mathbb{C}^{*}\right)^{n}$ we set $\mathbf{z}^{\mathbf{k}}=\prod_{i} z_{i}^{k_{i}}$.

It is easy to see that $G(\Lambda)$ is an algebraic subgroup. The converse holds true: Every algebraic subgroup is equal to $G(\Lambda)$ for some lattice $\Lambda$; see Schmidt [18]. If $\Lambda$ is primitive, then $G(\Lambda)$ is connected, and in this case it is called a torus. 
4.2.2 Automorphisms of $\left(\mathbb{C}^{*}\right)^{n}$ An example of a torus of dimension $l$ is the standard $l$-torus $T=\left(\mathbb{C}^{*}\right)^{l} \times 1^{n-l} \subset\left(\mathbb{C}^{*}\right)^{n}$, which is $G\left(\Xi_{n-l}\right)$, where

$$
\Xi_{n-l}=\left\{\left(k_{1}, \ldots, k_{n}\right) \in \mathbb{Z}^{n} \mid k_{1}=\cdots=k_{l}=0\right\} .
$$

The following trick shows that each torus is isomorphic to the standard torus. For details, see [18].

For matrix $K \in G L_{n}(\mathbb{Z})$ with entries $\left(K_{i j}\right)_{i, j=1}^{n}$, one can define an automorphism $\varphi_{K}$ of $\left(\mathbb{C}^{*}\right)^{n}$ by

$$
\varphi_{K}\left(z_{1}, z_{2}, \ldots, z_{n}\right)=\left(\prod_{j=1}^{n} z_{j}^{K_{1} j}, \prod_{j=1}^{n} z_{j}^{K_{2} j}, \ldots, \prod_{j=1}^{n} z_{j}^{K_{n} j}\right) .
$$

For any lattice $\Lambda \subset \mathbb{Z}^{n}, \varphi_{K}(G(\Lambda))=G(K(\Lambda))$. When $\Lambda$ is a primitive lattice of rank $n-l$, there is $K \in G L_{n}(\mathbb{Z})$ such that $K(\Lambda)=\Xi_{n-l}$. Then $\varphi_{K}(G(\Lambda))$ is the standard $l$-torus.

4.2.3 Algebraic subgroups and character groups Fix generators $t_{1}, \ldots, t_{n}$ of $\mathbb{Z}^{n}$. We will write $\mathbb{Z}^{n}$ multiplicatively and use the identification $\mathbb{Z}\left[\mathbb{Z}^{n}\right]=\mathbb{Z}\left[t_{1}^{ \pm 1}, \ldots, t_{n}^{ \pm 1}\right]$.

Suppose $\Gamma \subset \mathbb{Z}^{n}$ is a lattice. Every element $\mathbf{z} \in G(\Gamma)$ defines a character $\chi_{\mathbf{z}}$ of the quotient group $A_{\Gamma}:=\mathbb{Z}^{n} / \Gamma$ via

$$
\chi_{\mathbf{z}}\left(t_{1}^{k_{1}} \cdots t_{n}^{k_{n}}\right)=\mathbf{z}^{\mathbf{k}}, \quad \text { where } \mathbf{k}=\left(k_{1}, \ldots, k_{n}\right) .
$$

Conversely, every character of $A_{\Gamma}$ arises in this way. Thus one can identify $G(\Gamma)$ with the Pontryagin dual $\hat{A}_{\Gamma}$ via $\mathbf{z} \rightarrow \chi_{\mathbf{z}}$.

We will write $e_{\mathbf{z}}$ for the idempotent $e_{\chi_{\mathbf{z}}}$, and the decomposition (10), with $\Gamma$ having maximal rank, now becomes

$$
\mathbb{C}\left[A_{\Gamma}\right]=\bigoplus_{\mathbf{z} \in G(\Gamma)} \mathbb{C} e_{\mathbf{z}}
$$

\subsection{Torsion points in $\mathbb{Q}$-algebraic sets}

4.3.1 Torsion points With respect to the usual multiplication, $\mathbb{C}^{*}:=\mathbb{C} \backslash\{0\}$ is an abelian group, and so is the direct product $\left(\mathbb{C}^{*}\right)^{n}$. The subgroup of torsion elements of $\mathbb{C}^{*}$, denoted by $\mathbb{U}$, is the group of roots of unity, and $\mathbb{U}^{n}$ is the torsion subgroup of $\left(\mathbb{C}^{*}\right)^{n}$.

If $\Gamma \subset \mathbb{Z}^{n}$ is a lattice of maximal rank, then $G(\Gamma)$ is finite, and $G(\Gamma) \subset \mathbb{U}^{n}$. 
4.3.2 Torsion points and torsion coset A torsion coset is any set of the form $\mathbf{z} G(\Lambda)$, where $\mathbf{z} \in \mathbb{U}^{n}$ and $\Lambda \subset \mathbb{Z}^{n}$ is primitive, ie, $G(\Lambda)$ is a torus.

Suppose $Z \subset \mathbb{C}^{n}$. A torsion coset $X \subset Z$ is called a maximal torsion coset in $Z$ if it is not a proper subset of any torsion coset in $Z$.

The following fact, known as the Manin-Mumford theory for torsion points, is well known; see Laurent [8] and Schmidt [18].

Proposition 4.1 [8] Suppose $Z \subset \mathbb{C}^{n}$ is an algebraic closed set defined over $\mathbb{Q}$. There are in total only a finite number of maximal torsion cosets $X_{j} \subset Z, j=1, \ldots, q$. A torsion point $\mathbf{z} \in \mathbb{U}^{n}$ belongs to $Z$ if and only if $z \in X_{j}$ for some $j$, ie,

$$
Z \cap \mathbb{U}^{n}=\bigcup_{j=1}^{q}\left(X_{j} \cap \mathbb{U}^{n}\right) .
$$

Remark 4.1 The theory of torsion points in algebraic sets was first used in Clair and Whyte [3] to study the growth of the free parts of homology groups in abelian coverings.

4.3.3 $\mathbb{Q}$-closure Let $\operatorname{Gal}(\mathbb{C} / \mathbb{Q})$ be the set of all field automorphisms of $\mathbb{C}$. For $\mathbf{z}=$ $\left(z_{1}, \ldots, z_{n}\right)$, a Galois conjugate of $\mathbf{z}$ is any point of the form $\sigma(\mathbf{z})=\left(\sigma\left(z_{1}\right), \ldots, \sigma\left(z_{n}\right)\right)$, where $\sigma \in \operatorname{Gal}(\mathbb{C} / \mathbb{Q})$.

Suppose $X \subset \mathbb{C}^{n}$. Define the $\mathbb{Q}$-closure of $X$ by

$$
\operatorname{cl}_{\mathbb{Q}}(X):=\bigcup_{\sigma \in \operatorname{Gal}(\mathbb{C} / \mathbb{Q})} \sigma(X) .
$$

If $X \subset Z$, where $Z \subset \mathbb{C}^{n}$ is an algebraic set defined over $\mathbb{Q}$, then $\operatorname{cl}_{\mathbb{Q}}(X) \subset Z$.

If $\mathbf{z} \in \mathbb{U}^{n}$ is a torsion point, then the $\mathbb{Q}$-closure of $\{\mathbf{z}\}$, also denoted by $\operatorname{cl}_{\mathbb{Q}}(\mathbf{z})$, consists of a finite number of torsion points. One can prove that if torsion order of $\mathbf{z}$ is $k$, then $\left|\mathrm{cl}_{\mathbb{Q}}(\mathbf{z})\right|=\phi(k)$, where $\phi$ is the Euler totient function, though we do not need this result.

Lemma 4.2 Let $X \subset\left(\mathbb{C}^{*}\right)^{n}$ be a torsion coset. Then there exists a torsion point $\mathbf{u} \in \mathbb{U}^{n}$ and a primitive lattice $\Lambda \subset \mathbb{Z}^{n}$ such that

$$
\operatorname{cl}_{\mathbb{Q}}(X)=\bigsqcup_{\mathbf{z} \in \operatorname{cl}_{\mathbb{Q}}(\mathbf{u})} \mathbf{z} G(\Lambda)
$$


Proof By definition, there is a torus $T$ of dimension $l$ and a torsion point $\mathbf{u}^{\prime}$ such that $X=\mathbf{u}^{\prime} T$. Using an automorphism as described in Section 4.2.2, we can assume that $T$ is the standard $l$-torus, $T:=\left(\mathbb{C}^{*}\right)^{l} \times 1^{n-l}$. Let $\mathbf{u} \in \mathbb{U}^{n}$ be the point whose first $l$-coordinates are 1 , and the $j^{\text {th }}$ coordinate is the same as that of $\mathbf{u}^{\prime}$ for $j>l$. Alternatively, $\mathbf{u}$ is the only intersection point of $X$ and $1^{l} \otimes\left(\mathbb{C}^{*}\right)^{n-l}$.

Then $\mathbf{u} T=\mathbf{u}^{\prime} T=X$. Any Galois conjugate of $\mathbf{u}$ is in $1^{l} \otimes\left(\mathbb{C}^{*}\right)^{n-l}$. If $\mathbf{z}, \mathbf{z}^{\prime}$ are two distinct Galois conjugates of $\mathbf{u}$, then $\mathbf{z}^{-1} \mathbf{z}^{\prime} \in 1^{l} \otimes\left(\mathbb{C}^{*}\right)^{n-l}$, and hence $\mathbf{z}^{-1} \mathbf{z}^{\prime} \notin G(\Lambda)$. It follows easily that $\mathrm{cl}_{\mathbb{Q}}(X)=\operatorname{cl}_{\mathbb{Q}}(\mathbf{u} T)=\bigsqcup_{\mathbf{z} \in \mathrm{cl}_{\mathbb{Q}}(\mathbf{u})} \mathbf{z} T$.

\section{A growth rate estimate}

In this section we prove Proposition 5.4, establishing a crucial growth estimate of volumes of subspaces depending on a torsion coset $X$ and a lattice $\Gamma \subset \mathbb{Z}^{n}$ of maximal rank.

\subsection{Settings and notations}

Throughout this section fix a torsion coset $X \subset\left(\mathbb{C}^{*}\right)^{n}, X \neq\left(\mathbb{C}^{*}\right)^{n}$, and a $k \times l$ matrix $\mathcal{D}$ with entries in $\mathbb{Z}\left[\mathbb{Z}^{n}\right]=\mathbb{Z}\left[t_{1}^{ \pm 1}, \ldots, t_{n}^{ \pm 1}\right]$.

By right multiplication, we consider $\mathcal{D}$ as a $\mathbb{C}\left[\mathbb{Z}^{n}\right]$-morphism

$$
\mathcal{D}: \mathbb{C}\left[\mathbb{Z}^{n}\right]^{k} \rightarrow \mathbb{C}\left[\mathbb{Z}^{n}\right]^{l} .
$$

Suppose $\Gamma \leq \mathbb{Z}^{n}$ is a lattice of maximal rank. In what follows we fix $X, \mathcal{D}$ but vary $\Gamma$. Let $A=A_{\Gamma}:=\mathbb{Z}^{n} / \Gamma$, a finite abelian group. Equip $\mathbb{C}[A]$ with the structure of a based Hermitian space as in Section 4.1. The fundamental lattice of $\mathbb{C}[A]$ is $\mathbb{Z}[A]$.

The map $\mathcal{D}$ in (15) descends to an integral $\mathbb{C}[A]$-morphism

$$
\mathcal{D}_{\Gamma}: \mathbb{C}[A]^{k} \rightarrow \mathbb{C}[A]^{l} .
$$

By Lemma 4.2, there is a primitive lattice $\Lambda \leq \mathbb{Z}^{n}$ and a torsion point $\mathbf{u} \in \mathbb{U}^{n}$ such that

$$
\operatorname{cl}_{\mathbb{Q}}(X)=\bigsqcup_{j=1}^{r} \mathbf{u}_{j} G(\Lambda),
$$

where $\left\{\mathbf{u}_{j}, j=1 \ldots, r\right\}$ is the set of all Galois conjugates of $\mathbf{u}$. Since $X \neq\left(\mathbb{C}^{*}\right)^{n}, \Lambda$ is not the trivial group, $\Lambda \neq\{0\}$.

Let $B=B_{\Gamma, \Lambda}:=(\Gamma+\Lambda) / \Gamma$. Then $B$ is a subgroup of $A=\mathbb{Z}^{n} / \Gamma$. 


\subsection{Decomposition of $\mathcal{D}_{\Gamma}$ and norm of $\mathcal{D}_{\Gamma}$}

Recall that $\hat{A}=G(\Gamma)$ and we have the decomposition (13) of $A$-module

$$
\mathbb{C}[A]=\bigoplus_{\mathbf{z} \in \widehat{A}} \mathbb{C} e_{\mathbf{z}} .
$$

For each $\mathbf{z} \in \widehat{A}=G(\Gamma), \mathcal{D}$ induces a $\mathbb{C}[A]$-map

$$
\mathcal{D}(\mathbf{z}):\left(\mathbb{C} e_{\mathbf{z}}\right)^{k} \rightarrow\left(\mathbb{C} e_{\mathbf{z}}\right)^{l} .
$$

Here $\mathcal{D}(\mathbf{z})$ is simply the $k \times l$ matrix with entries in $\mathbb{C}$, obtained by evaluating $\mathcal{D}$ at z. (Recall that each entry of $\mathcal{D}$ is a Laurent polynomial in $n$ variables, and one can evaluate such a Laurent polynomial at any point $\mathbf{z} \in\left(\mathbb{C}^{*}\right)^{n}$.) We have:

$$
\mathcal{D}_{\Gamma}=\bigoplus_{\mathbf{z} \in \hat{A}} \mathcal{D}(\mathbf{z})
$$

It follows that:

$$
\left\|\mathcal{D}_{\Gamma}\right\|=\max _{\mathbf{z} \in \widehat{A}}\|\mathcal{D}(\mathbf{z})\|
$$

It is easy to see that the norm of any $k \times l$ matrix is less than or equal to $k l$ times the sum of the absolute values of all the entries.

For a Laurent polynomial $f \in \mathbb{Z}\left[\mathbb{Z}^{n}\right]$ let the $\ell^{1}$-norm of $f$ be the sum of the absolute values of all the coefficients of $f$. Let $\|\mathcal{D}\|_{1}$ be the sum of the $\ell^{1}$-norms of all of its entries. Then we have $\|\mathcal{D}(\mathbf{z})\| \leq k l\|\mathcal{D}\|_{1}$ because each component of $\mathbf{z}$ has absolute value 1 . Thus we have the following uniform upper bound for $\mathcal{D}_{\Gamma}$ :

$$
\left\|\mathcal{D}_{\Gamma}\right\| \leq k l\|\mathcal{D}\|_{1}
$$

\subsection{Integral decomposition of $\mathcal{D}_{\Gamma}$ along $X$}

For each $\mathbf{z} \in G(\Gamma)$, the 1 -dimensional vector space $\mathbb{C} e_{\mathbf{z}}$ is in general not defined over $\mathbb{Q}$. However, its $\mathbb{Q}$-closure is defined over $\mathbb{Q}$.

Consider the following $\mathbb{C}[A]$-submodule $\alpha(\Gamma, X)$ of $\mathbb{C}[A]$ :

$$
\alpha(\Gamma, X):=\bigoplus_{\mathbf{z} \in \hat{A} \cap c_{\mathbb{Q}}(X)} \mathbb{C} e_{\mathbf{z}} \subset \mathbb{C}[A]=\bigoplus_{\mathbf{z} \in \hat{A}} \mathbb{C} e_{\mathbf{z}}
$$

Since the set $\hat{A} \cap \mathrm{cl}_{\mathbb{Q}}(X)$ is closed under Galois conjugations, $\alpha(\Gamma, X)$ is defined over $\mathbb{Q}$. The orthogonal complement of $\alpha(\Gamma, X)$ is:

$$
\alpha^{\perp}(\Gamma, X):=\bigoplus_{\mathbf{z} \in \hat{A} \backslash \operatorname{cl}_{\mathbb{Q}}(X)} \mathbb{C} e_{\mathbf{z}}
$$


We have $\mathbb{C}[A]=\alpha(\Gamma, X) \oplus \alpha^{\perp}(\Gamma, X)$, and each of $\left\{\alpha(\Gamma, X), \alpha^{\perp}(\Gamma, X)\right\}$ is an ideal of $\mathbb{C}[A]$, or an $A$-subspace of $\mathbb{C}[A]$.

The $A$-morphism $\mathcal{D}_{\Gamma}$ restricts to $A$-morphisms

$$
\mathcal{D}_{\Gamma, X}: \alpha(\Gamma, X)^{k} \rightarrow \alpha(\Gamma, X)^{l} \quad \text { and } \quad \mathcal{D}_{\Gamma, X}^{\perp}:\left(\alpha^{\perp}(\Gamma, X)\right)^{k} \rightarrow\left(\alpha^{\perp}(\Gamma, X)\right)^{l},
$$

which are integral. We have $\mathcal{D}_{\Gamma}=\mathcal{D}_{\Gamma, X} \oplus \mathcal{D}_{\Gamma, X}^{\perp}$.

\subsection{Projection onto $\alpha_{\Gamma, X}$}

Lemma 5.1 If $G(\Gamma) \cap X \neq \varnothing$, then

$$
\operatorname{dim}_{\mathbb{C}}\left(\alpha_{\Gamma, X}\right)=r|A| /|B|
$$

and the orthogonal projection from $\mathbb{C}[A]$ onto $\alpha_{\Gamma, X}$ is given by the idempotent

$$
N_{X}:=\frac{1}{|B|} \sum_{j=1}^{r} \sum_{b \in B} \mathbf{u}_{j}\left(b^{-1}\right) b .
$$

Proof Since $\hat{A}=G(\Gamma)$ is defined over $\mathbb{Q}$, if it intersects $X$, then it intersects every component $\mathbf{u}_{j} G(\Lambda)$ of the decomposition (16) of $\mathrm{cl}_{\mathbb{Q}}(X)$. Let $\mathbf{u}_{j}^{\prime} \in G(\Gamma) \cap \mathbf{u}_{j} G(\Lambda)$. Since $G(\Lambda)$ is a subgroup of $\left(\mathbb{C}^{*}\right)^{n}$ and $\mathbf{u}_{j}^{\prime} \in \mathbf{u}_{j} G(\Lambda)$, we have $\mathbf{u}_{j} G(\Lambda)=\mathbf{u}_{j}^{\prime} G(\Lambda)$ and

$$
\mathbf{u}_{j}^{-1} \mathbf{u}_{j}^{\prime} \in G(\Lambda) .
$$

Since $G(\Gamma)$ is a subgroup and $\mathbf{u}_{j}^{\prime} \in G(\Gamma)$, we have $G(\Gamma)=\mathbf{u}_{j}^{\prime} G(\Gamma)$, and hence

$$
G(\Gamma) \cap \mathbf{u}_{j} G(\Lambda)=\mathbf{u}_{j}^{\prime}[G(\Gamma) \cap G(\Lambda)]=\mathbf{u}_{j}^{\prime}[G(\Gamma+\Lambda)] .
$$

From the above identity and the decomposition (16) we have

$$
G(\Gamma) \cap \operatorname{cl}_{\mathbb{Q}}(X)=\bigsqcup_{j=1}^{r} \mathbf{u}_{j}^{\prime}[G(\Gamma+\Lambda)] .
$$

Let $s=|A| /|B|$, which is the cardinality of the quotient group $A / B=\mathbb{Z}^{n} /(\Gamma+\Lambda)$. Then $|G(\Gamma+\Lambda)|=s$, since $G(\Gamma+\Lambda)$ is the Pontryagin dual of $A / B=\mathbb{Z}^{n} /(\Gamma+\Lambda)$. From (21) we have $\left|\hat{A} \cap \mathrm{cl}_{\mathbb{Q}}(X)\right|=r s$. Hence $\operatorname{dim}_{\mathbb{C}}\left(\alpha_{\Gamma, X}\right)=r s=r|A| /|B|$. This proves (18).

For each element of the quotient group $A / B$ choose a lift in $A$, and denote by $C$ the set of all such lifts. We assume that the chosen lift of the trivial element is $e$. Then

$$
A=\{b c \mid b \in B, c \in C\} .
$$


From (12), for every $c \in C$,

$$
\sum_{\mathbf{z} \in G(\Gamma+\Lambda)} \mathbf{z}(c)= \begin{cases}0 & \text { if } c \neq e, \\ |C| & \text { if } c=e .\end{cases}
$$

Recall that for $\mathbf{z} \in \hat{A}=G(\Lambda), e_{\mathbf{z}}$ is an idempotent in $\mathbb{C}[A]$, and $e_{\mathbf{z}} e_{\mathbf{z}^{\prime}}=0$ if $\mathbf{z} \neq \mathbf{z}^{\prime}$. It follows that the projection onto $\alpha_{\Gamma, X}$ is given by $N_{X}=\sum_{\mathbf{z} \in \hat{A} \cap c_{\mathbb{Q}}(X)} e_{\mathbf{z}}$.

Using (11), we have

$$
\begin{aligned}
N_{X} & =\frac{1}{|A|} \sum_{\mathbf{z} \in \hat{A} \cap \mathbf{c}_{\mathbb{Q}}(X)} \sum_{a \in A} \mathbf{z}\left(a^{-1}\right) a \\
& =\frac{1}{|A|} \sum_{\mathbf{z} \in \hat{A} \cap \mathbf{l}_{\mathbb{Q}}(X)} \sum_{b \in B} \sum_{c \in C} \mathbf{z}\left(b^{-1} c^{-1}\right) b c \\
& =\frac{1}{|B||C|} \sum_{\mathbf{z} \in \hat{A} \cap \mathrm{cl} \mathbb{Q}(X)}\left[\sum_{b \in B} \mathbf{z}\left(b^{-1}\right) b\right]\left[\sum_{c \in C} \mathbf{z}\left(c^{-1}\right) c\right] \\
& =\frac{1}{|B||C|} \sum_{j=1}^{r} \sum_{\mathbf{z} \in G(\Gamma+\Lambda)}\left[\sum_{b \in B} \mathbf{z}\left(b^{-1}\right) \mathbf{u}_{j}^{\prime}\left(b^{-1}\right) b\right]\left[\sum_{c \in C} \mathbf{z}\left(c^{-1}\right) \mathbf{u}_{j}^{\prime}\left(c^{-1}\right) c\right] \text { by }(21) \\
& =\frac{1}{|B||C|} \sum_{j=1}^{r} \sum_{\mathbf{z} \in G(\Gamma+\Lambda)}\left[\sum_{b \in B} \mathbf{u}_{j}^{\prime}\left(b^{-1}\right) b\right]\left[\sum_{c \in C} \mathbf{z}\left(c^{-1}\right) \mathbf{u}_{j}^{\prime}\left(c^{-1}\right) c\right] \\
& =\frac{1}{|B||C|} \sum_{j=1}^{r}\left[\sum_{b \in B} \mathbf{u}_{j}^{\prime}\left(b^{-1}\right) b \sum_{\mathbf{z} \in G(\Gamma+\Lambda)} \sum_{c \in C} \mathbf{z}\left(c^{-1}\right) \mathbf{u}_{j}^{\prime}\left(c^{-1}\right) c\right. \\
& =\frac{1}{|B|} \sum_{j=1}^{r} \sum_{b \in B} \mathbf{u}_{j}^{\prime}\left(b^{-1}\right) b \quad \text { by }(22) \\
& =\frac{1}{|B|} \sum_{j=1}^{r} \sum_{b \in B} \mathbf{u}_{j}\left(b^{-1}\right) b \quad \text { by }(20) .
\end{aligned}
$$

This completes the proof of the lemma.

\subsection{Upper bound for the volume of $\alpha_{\Gamma, X}$}

Lemma 5.2 One has:

$$
\operatorname{vol}\left(\alpha_{\Gamma, X}\right) \leq(r|B|)^{r|A| /|B|}
$$


Proof We assume that $G(\Gamma) \cap X \neq \varnothing$, because otherwise $\alpha_{\Gamma, X}=0$, and the statement is trivial.

From (19),

$$
|B| N_{X}=\sum_{b \in B}\left(\sum_{j=1}^{r} \mathbf{u}_{j}\left(b^{-1}\right)\right) b \in \mathbb{Z}[B] .
$$

If $a \in A$, then from (19) we have

$$
|B| N_{X} a=\sum_{j=1}^{r} \sum_{b \in B} \mathbf{u}_{j}\left(b^{-1}\right) b a .
$$

Because $\left\|\mathbf{u}_{j}\left(b^{-1}\right) b a\right\|=1$, we see that $|B| N_{X} a$ has length $\leq r|B|$.

By Lemma 5.1, $\alpha(\Gamma, X)$ has dimension $r s$. Since $A$ spans $\mathbb{C}[A]$ and $N_{X}$ is the projection onto $\alpha_{\Gamma, X}$, the set $\left\{N_{X} a \mid a \in A\right\}$ spans $\alpha_{\Gamma, X}$. Hence, there are $a_{1}, \ldots, a_{r s} \in A$ such that $\left\{N_{X} a_{j}, j=1, \ldots, r s\right\}$ is a basis of the vector space $\alpha_{\Gamma, X}$. Since $|B| N_{X} a_{j} \in$ $\mathbb{Z}[A]$ is integral, $\operatorname{vol}\left(\alpha_{\Gamma, X}\right)$ is less than or equal to the volume of the parallelepiped spanned by $\left\{|B| N_{X} a_{j}, j=1, \ldots, r s\right\}$. The length of each $|B| N_{X} a_{j}$ is at most $r|B|$. It follows that $\operatorname{vol}(\alpha) \leq(r|B|)^{r s}=(r|B|)^{r|A| /|B|}$.

\subsection{Growth of $|B|$}

The following statement has been used in [9]. This is the place we use the assumption $\langle\Gamma\rangle \rightarrow \infty$ and $\Lambda \neq 0$.

Lemma 5.3 Suppose $\Lambda$ is a non-trivial lattice. Then

$$
\lim _{\langle\Gamma\rangle \rightarrow \infty}|B|=\infty
$$

Proof For the length of $x \in \pi=\mathbb{Z}^{n}$ (in the definition of $\langle\Gamma\rangle$ ) we will use the standard metric $|x|$ derived from the Hermitian structure.

Fix an element $x \in \Lambda, x \neq 0$, and look at the degree of $x$ in $B=(\Lambda+\Gamma) / \Gamma$. If $M|x|<\langle\Gamma\rangle$ for some positive integer $M$, then $M|x|$ does not belong to $\Gamma$ by the definition of $\langle\Gamma\rangle$, and hence $M x$ is not 0 in $B=(\Lambda+\Gamma) / \Gamma$. This means the cyclic subgroup of $B$ generated by $x$ has order at least $\langle\Gamma\rangle /|x|$. It follows that $|B| \geq\langle\Gamma\rangle /|x|$. Hence $\lim _{\langle\Gamma\rangle \rightarrow \infty}|B|=\infty$. 


\subsection{Growth of $\operatorname{ker}\left(\mathcal{D}_{\Gamma, X}\right)$}

Proposition 5.4 For a fixed torsion coset $X \subset\left(\mathbb{C}^{*}\right)^{n}$ and a $k \times l$ matrix $\mathcal{D}$ with entries in $\mathbb{Z}\left[\mathbb{Z}^{n}\right]$, we have

$$
\lim _{\langle\Gamma\rangle \rightarrow \infty,\left|\mathbb{Z}^{n}: \Gamma\right|<\infty} \frac{\ln \left[\operatorname{vol}\left(\operatorname{ker}\left(\mathcal{D}_{\Gamma, X}\right)\right)\right]}{\left|\mathbb{Z}^{n}: \Gamma\right|}=0 .
$$

Proof To simplify notation we will write $\alpha=\alpha_{\Gamma, X}$. By (6),

$$
\operatorname{vol}\left(\operatorname{ker} \mathcal{D}_{\Gamma, X}\right) \operatorname{vol}\left(\operatorname{Im} \mathcal{D}_{\Gamma, X}^{(\mathbb{Z})}\right)=\operatorname{det}^{\prime} \mathcal{D}_{\Gamma, X}(\operatorname{vol}(\alpha))^{k}
$$

Because $\operatorname{vol}\left(\operatorname{Im} \mathcal{D}_{\Gamma, X}^{(\mathbb{Z})}\right) \geq 1$, we have:

$$
\begin{aligned}
\operatorname{vol}\left(\operatorname{ker} \mathcal{D}_{\Gamma, X}\right) & \leq \operatorname{det}^{\prime} \mathcal{D}_{\Gamma, X}(\operatorname{vol}(\alpha))^{k} \\
& \leq\left\|\mathcal{D}_{\Gamma, X}\right\|^{\operatorname{dim}(\alpha)}(\operatorname{vol}(\alpha))^{k} \quad \text { by (4) } \\
& \leq\left\|\mathcal{D}_{\Gamma, X}\right\|^{r|A| /|B|}(r|B|)^{k r|A| /|B|} \quad \text { by (18) and (23) }
\end{aligned}
$$

Since $\mathcal{D}_{\Gamma, X}$ is a restriction of $\mathcal{D}_{\Gamma}$ on a subspace, we have

$$
\left\|\mathcal{D}_{\Gamma, X}\right\| \leq\left\|\mathcal{D}_{\Gamma}\right\| \leq k l\|\mathcal{D}\|_{1},
$$

where the second inequality is (17).

It follows that

$$
\operatorname{vol}\left(\operatorname{ker} \mathcal{D}_{\Gamma, X}\right) \leq\left(k l\|\mathcal{D}\|_{1}(r|B|)^{k}\right)^{r|A| /|B|},
$$

and

$$
\frac{\ln \left[\operatorname{vol}\left(\operatorname{ker}\left(\mathcal{D}_{\Gamma, X}\right)\right)\right]}{|A|} \leq \frac{r \ln \left(k l\|\mathcal{D}\|_{1}(r|B|)^{k}\right)}{|B|} .
$$

Because $|B| \rightarrow \infty$ as $\langle\Gamma\rangle \rightarrow \infty$ by (24), the right hand side of (25) goes to 0 as $\langle\Gamma\rangle \rightarrow \infty$.

\section{Proofs of Theorems 1 and 2}

It is clear that Theorem 1 follows from Theorem 2 and Identity (7). We will prove Theorem 2 in this section. 


\subsection{Preliminaries}

Recall that $\mathcal{C}$ is a finitely generated based free $\mathbb{Z}\left[\mathbb{Z}^{n}\right]$-complex

$$
0 \rightarrow C_{m} \stackrel{\partial_{m}}{\longrightarrow} C_{m-1} \stackrel{\partial_{m-1}}{\longrightarrow} C_{m-2} \longrightarrow \cdots \stackrel{\partial_{2}}{\longrightarrow} C_{1} \stackrel{\partial_{1}}{\longrightarrow} C_{0} \rightarrow 0 .
$$

Using the bases of $C_{j}$ 's we identify $C_{j}$ with $\mathbb{Z}\left[\mathbb{Z}^{n}\right]^{b_{j}}$ and $\partial_{j}$ with a $b_{j} \times b_{j-1}$ matrix with entries in $\mathbb{Z}\left[\mathbb{Z}^{n}\right]=\mathbb{Z}\left[t_{1}^{ \pm 1}, \ldots, t_{n}^{ \pm 1}\right]$.

For $f=\sum a_{j} g_{j} \in \mathbb{Z}\left[\mathbb{Z}^{n}\right]$, where $a_{j} \in \mathbb{Z}$ and $g_{j} \in \mathbb{Z}^{n}$, let $f^{*}=\sum a_{j} g_{j}^{-1}$. If $f$ acts on $\ell^{2}\left(\mathbb{Z}^{n}\right)$, the Hilbert space with basis $\mathbb{Z}^{n}$, by multiplication, then $f^{*}$ is the adjoint operator of $f$. As usual, for a matrix $O=\left(O_{i j}\right)$ with entries in $\mathbb{Z}\left[\mathbb{Z}^{n}\right]$, let the adjoint $O^{*}$ be defined by $\left(O^{*}\right)_{i j}=\left(O_{j i}\right)^{*}$.

According to the assumption of Theorem 2,

$$
H_{k}\left(\mathcal{C} \otimes_{\mathbb{Z}\left[\mathbb{Z}^{n}\right]} F\right)=0,
$$

where $F$ is the fractional field of $\mathbb{Z}\left[\mathbb{Z}^{n}\right]$. Let

$$
\mathcal{D}=\partial_{k}^{*} \partial_{k}+\partial_{k+1} \partial_{k+1}^{*}: C_{k} \rightarrow C_{k} .
$$

Then (26) is equivalent to the fact that $\mathcal{D}$ is an injective map, or that $\operatorname{det}(\mathcal{D}) \neq 0$. Here $\operatorname{det}(\mathcal{D})$ is the usual determinant of a square matrix with entries in a commutative ring. In our case $\operatorname{det}(\mathcal{D})$ is a Laurent polynomial in $t_{1}, \ldots, t_{n}$.

Let $Z$ be the zero set of the Laurent polynomial $\operatorname{det}(\mathcal{D})$, ie, $Z=\left\{\mathbf{z} \in \mathbb{C}^{n} \mid \operatorname{det}(\mathcal{D})(\mathbf{z})=\right.$ $0\}$. In other words, $Z$ is the set of $\mathbf{z} \in \mathbb{C}^{n}$ such that the square matrix $\mathcal{D}(\mathbf{z})$ is singular. Since $\operatorname{det}(\mathcal{D}) \neq 0, Z$ is not the whole $\mathbb{C}^{n}$.

For every subgroup $\Gamma \leq \mathbb{Z}^{n}, \mathcal{D}$ induces a map $\mathcal{D}_{\Gamma}: \mathbb{C}[A]^{b_{k}} \rightarrow \mathbb{C}[A]^{b_{k}}$, where $A=$ $\mathbb{Z}^{n} / \Gamma$.

\subsection{Growth of $\operatorname{vol}\left(\operatorname{ker}\left(\mathcal{D}_{\Gamma}\right)\right)$}

Proposition 6.1 In the above setting, we have

$$
\lim _{\langle\Gamma\rangle \rightarrow \infty,|\pi: \Gamma|<\infty} \frac{\ln \left[\operatorname{vol}\left(\operatorname{ker}\left(\mathcal{D}_{\Gamma}\right)\right)\right]}{|\pi: \Gamma|}=0 .
$$

Proof By Proposition 4.1, there are in total a finite number of maximal torsion cosets $X_{j} \subset Z$, sets $j=1, \ldots, q$, and

$$
Z \cap \mathbb{U}^{n}=\bigcup_{j=1}^{q}\left(X_{j} \cap \mathbb{U}^{n}\right) .
$$


Since $Z \neq \mathbb{C}^{n}$, none of the lattices associated to the torsion cosets $X_{j}$ is trivial.

Because $Z$ is defined over $\mathbb{Q}$, any Galois conjugate of $X_{j}$ is also a maximal torsion coset in $Z$, ie, among $\left\{X_{1}, \ldots, X_{q}\right\}$.

Since $G(\Gamma) \subset \mathbb{U}^{n}$, one has

$$
Z \cap G(\Gamma)=\left(Z \cap \mathbb{U}^{n}\right) \cap G(\Gamma)=\bigcup_{j=1}^{q}\left(X_{j} \cap \mathbb{U}^{n}\right) \cap G(\Gamma)=\bigcup_{j=1}^{q}\left(X_{j} \cap G(\Gamma)\right) .
$$

Because the Galois conjugates of the $X_{j}$ are among the $X_{j}$, we also have

$$
Z \cap G(\Gamma)=\bigcup_{j=1}^{q}\left(\operatorname{cl}_{\mathbb{Q}}\left(X_{j}\right) \cap G(\Gamma)\right)
$$

Let

$$
\alpha:=\bigoplus_{\mathbf{z} \in G(\Gamma) \cap Z} \mathbb{C} e_{\mathbf{z}}, \quad \alpha^{\perp}:=\bigoplus_{\mathbf{z} \in G(\Gamma) \backslash Z} \mathbb{C} e_{\mathbf{z}}
$$

Then $\mathbb{C}[A]=\alpha \oplus \alpha^{\perp}$, and each of $\left\{\alpha, \alpha^{\perp}\right\}$ is an $A$-subspace of $\mathbb{C}[A]$. The linear operator $\mathcal{D}_{\Gamma}$ restricts to

$$
\mathcal{D}_{\Gamma, \alpha}: \alpha^{b_{k}} \rightarrow \alpha^{b_{k}} \quad \text { and } \quad \mathcal{D}_{\Gamma, \alpha}^{\perp}:\left(\alpha^{\perp}\right)^{b_{k}} \rightarrow\left(\alpha^{\perp}\right)^{b_{k}},
$$

and

$$
\mathcal{D}_{\Gamma}=\mathcal{D}_{\Gamma, \alpha} \oplus \mathcal{D}_{\Gamma, \alpha}^{\perp}
$$

where

$$
\mathcal{D}_{\Gamma, \alpha}=\bigoplus_{\mathbf{z} \in G(\Gamma) \cap Z} \mathcal{D}(\mathbf{z}), \quad \mathcal{D}_{\Gamma, \alpha}^{\perp}=\bigoplus_{\mathbf{z} \in G(\Gamma) \backslash Z} \mathcal{D}(\mathbf{z}) .
$$

When $\mathbf{z} \notin Z, \mathcal{D}(\mathbf{z})$ is non-singular. It follows that $\mathcal{D}_{\Gamma, \alpha}^{\perp}$ is non-singular. Hence:

$$
\begin{aligned}
& \operatorname{ker}\left(\mathcal{D}_{\Gamma}\right)=\operatorname{ker}\left(\mathcal{D}_{\Gamma, \alpha}\right) \\
&=\bigoplus_{\mathbf{z} \in G(\Gamma) \cap Z} \operatorname{ker}(\mathcal{D}(\mathbf{z})) \quad \text { by }(29) \\
&=\sum_{j=1}^{q}\left(\bigoplus_{\mathbf{z} \in \mathrm{cl}_{\mathbb{Q}}}\left(X_{j}\right) \cap G(\Gamma)\right. \\
&\operatorname{ker}(\mathcal{D}(\mathbf{z}))) \quad \text { by }(28)
\end{aligned}
$$

From (28) we have

$$
\alpha=\sum_{j=1}^{q}\left[\bigoplus_{\mathbf{z} \in G(\Gamma) \cap \mathrm{cl} \mathbb{Q}\left(X_{j}\right)} \mathbb{C} e_{\mathbf{z}}\right]=\sum_{j=1}^{q} \alpha\left(\Gamma, X_{j}\right),
$$


where $\alpha\left(\Gamma, X_{j}\right)$ is defined as in Section 5.3. One also has

$$
\operatorname{ker}\left(\mathcal{D}_{\Gamma, X_{j}}\right)=\bigoplus_{\mathbf{z} \in G(\Gamma) \cap \mathrm{cl}_{\mathbb{Q}}}\left(X_{j}\right)
$$

From (30) and (31), one has

$$
\operatorname{ker}\left(\mathcal{D}_{\Gamma}\right)=\sum_{j=1}^{q} \operatorname{ker}\left(\mathcal{D}_{\Gamma, X_{j}}\right) .
$$

Hence, by (5),

$$
\operatorname{vol}\left(\operatorname{ker}\left(\mathcal{D}_{\Gamma}\right)\right) \leq \prod_{j=1}^{q} \operatorname{vol}\left(\operatorname{ker}\left(\mathcal{D}_{\Gamma, X_{j}}\right)\right) .
$$

Now (27) follows from the above inequality and Proposition 5.4.

\subsection{Proof of Theorem 2}

Applying Proposition 3.1 to the $\mathbb{Z}$-complex $\mathcal{E}=\mathcal{C}_{\Gamma}$, we get

$$
\left.\operatorname{vol}\left(\operatorname{ker}\left(\mathcal{D}_{\Gamma}\right)\right) \geq R_{k}\left(\mathcal{C}_{\Gamma}\right)\right) \geq \frac{1}{\operatorname{vol}\left(\operatorname{ker}\left(\mathcal{D}_{\Gamma}\right)\right)} .
$$

Thus we have

$$
\frac{\ln \left(\operatorname{vol}\left(\operatorname{ker}\left(\mathcal{D}_{\Gamma}\right)\right)\right)}{\left|\mathbb{Z}^{n}: \Gamma\right|} \geq \frac{\ln \left(R_{k}\left(\mathcal{C}_{\Gamma}\right)\right)}{\left|\mathbb{Z}^{n}: \Gamma\right|} \geq-\frac{\ln \left(\operatorname{vol}\left(\operatorname{ker}\left(\mathcal{D}_{\Gamma}\right)\right)\right)}{\left|\mathbb{Z}^{n}: \Gamma\right|} .
$$

By Proposition 6.1, the limits of the two boundary terms, when $\langle\Gamma\rangle \rightarrow \infty$ and $\mid \mathbb{Z}^{n}$ : $\Gamma \mid<\infty$, are 0 . Hence we also have

$$
\lim _{\substack{\langle\Gamma\rangle \rightarrow \infty,\left|\mathbb{Z}^{n}: \Gamma\right|<\infty}} \frac{\ln \left(R_{k}(\Gamma)\right)}{\left|\mathbb{Z}^{n}: \Gamma\right|}=0 .
$$

This completes the proof of Theorem 2 .

\section{References}

[1] N Bergeron, A Venkatesh, The asymptotic growth of torsion homology for arithmetic groups, J. Inst. Math. Jussieu 12 (2013) 391-447 MR3028790

[2] D Bertrand, Duality on tori and multiplicative dependence relations, J. Austral. Math. Soc. Ser. A 62 (1997) 198-216 MR1433209

[3] B Clair, K Whyte, Growth of Betti numbers, Topology 42 (2003) 1125-1142 MR1978050 
[4] G Elek, Abelian coverings, J. Funct. Anal. 141 (1996) 365-373 MR1418511

[5] S Friedl, N Jackson, Approximations to the volume of hyperbolic knots arXiv: 1102.3742

[6] F González-Acuña, H Short, Cyclic branched coverings of knots and homology spheres, Rev. Mat. Univ. Complut. Madrid 4 (1991) 97-120 MR1142552

[7] J A Hillman, M Sakuma, On the homology of finite abelian coverings of links, Canad. Math. Bull. 40 (1997) 309-315 MR1464839

[8] M Laurent, Équations diophantiennes exponentielles, Invent. Math. 78 (1984) 299-327 MR767195

[9] T T Q Lê, Homology torsion growth and Mahler measure, to appear in Comment. Math. Helv. arXiv: 1010.4199

[10] T T Q Lê, Hyperbolic volume, Mahler measure, and homology growth, talk at Columbia University (2009) Available at http://www.math.columbia.edu/ volconf09/ notes/leconf.pdf

[11] W Lück, Approximating $L^{2}$-invariants by their finite-dimensional analogues, Geom. Funct. Anal. 4 (1994) 455-481 MR1280122

[12] W Lück, $L^{2}$-invariants: theory and applications to geometry and $K$-theory, Ergeb. Math. Grenzgeb. 44, Springer, Berlin (2002) MR1926649

[13] J P Mayberry, K Murasugi, Torsion-groups of abelian coverings of links, Trans. Amer. Math. Soc. 271 (1982) 143-173 MR648083

[14] J W Milnor, Infinite cyclic coverings, from: "Conference on the Topology of Manifolds”, (J G Hocking, editor), Prindle, Weber \& Schmidt, Boston (1968) 115-133 MR0242163

[15] J Porti, Mayberry-Murasugi's formula for links in homology 3-spheres, Proc. Amer. Math. Soc. 132 (2004) 3423-3431 MR2073320

[16] J Raimbault, Exponential growth of torsion in abelian coverings, Algebr. Geom. Topol. 12 (2012) 1331-1372 MR2966689

[17] R Riley, Growth of order of homology of cyclic branched covers of knots, Bull. London Math. Soc. 22 (1990) 287-297 MR1041145

[18] W Schmidt, Heights of points on subvarieties of $\mathbf{G}_{m}^{n}$, from: "Number theory", (V K Murty, M Waldschmidt, editors), Contemp. Math. 210, Amer. Math. Soc., Providence, RI (1998) 97-99 MR1478487

[19] J-P Serre, Linear representations of finite groups, Graduate Texts in Mathematics 42, Springer, New York (1977) MR0450380

[20] D S Silver, S G Williams, Mahler measure, links and homology growth, Topology 41 (2002) 979-991 MR1923995 
[21] V Turaev, Introduction to combinatorial torsions, Lectures in Mathematics ETH Zürich, Birkhäuser, Basel (2001) MR1809561

School of Mathematics, Georgia Institute of Technology Atlanta, GA 30332-0160, USA

letu@math.gatech.edu

http://people.math.gatech.edu/ letu/

Received: 4 January 2013 Revised: 22 March 2013 